60. Sur les Bupleurum Odontites L. part. et opacum. (Ces.) Lge. (Ibid., LV, 437-439, 1908.)

61. Les Érables de Savoie. (Ibid., LVI, 383-389, 1909.) - Travail inachevé à cause d'une documenlation que l'auteur avait reconnue insuffisante, repris $a b$ ovo l'année suivante $\left(\mathbf{n}^{\circ} 64\right)$.

62. Une Scille nouvelle d'Algerie. (Ibid., LVI, 477-478, 1909.)

63. Sur les Pedicularis de la Saroie. (Ibid., LVI, $499-502,1909$.

64. Revision des Érables de la Savoie. (Ibid., LVII, 10-18, 39-47, pl. 1-IV, 1910.)

63̈. Un dernier mot sur les Pédiculaires de la Savoie. (Ibid., LVII, 89-90, 1910.)

Les deux communications suivantes sont ensuite présentées :

\title{
Observations sur la flore des Alpes maritimes
}

\section{PAR M. AUG. CHEVALIER.}

Mon affectation à un dépôt de troupes coloniales, m'a valu de séjourner pendant les deux années 1915 et 1916 a Menton, la localité certainement la plus chaude de la France continentale. J'ai consacré à des herborisations les rares loisirs que me laissaient mes occupations militaires. Dans la région comprise entre Nice et Menton sur le littoral, et s'étendant dans l'intérieur jusqu'au mont Authion $(2080 \mathrm{~m}$. alt.), j'ai recueilli plusieurs centaines d'espèces ou races de Phanérogames. J'ai été amené à faire ainsi sur la flore de ces régions quelques observations que je crois utile de résumer brièvement ici.

On sait que la flore des Alpes maritimes est connue surtout grâce aux recherches de Ardoino, auteur de la Flore analytique des Alpes maritimes (1867), de M. Burnat et de ses collaborateurs, principalement J. Briquet et F. Cavillier, de nos collègues MM. J. Arbost et le Commandant A. Saint-Yves. Elle présente un certain nombre de particularités intéressantes :

$1^{\circ}$ De tous les départements continentaux français, celui des Alpes-Maritimes, l'un des plus petits par l'étendue, est de beaucoup le plus riche au point de vue floristique aussi bien par le nombre des espèces de Phanérogames qui y sont spontanées que par la très grande quantité d'espèces exotiques qui 
A. CHEVAlier. - SUR LA FloRE DES ALPES MARITIMES.

y sont parfaitement acclimatées dans les parcs et les jardins et qui tout le long du littoral modifient complètement l'aspect du paysage.

En 1867, Ardoino comptait 2466 Phanérogames spontanées sur une aire débordant, il est vrai, un peu sur le département du Var à l'Ouest et sur l'Italie à l'Est. En tenant compte des découvertes faites depuis cinquante ans il n'est pas exagéré de supposer que le nombre des espèces nettement caractérisées qui y sont actuellement connues à l'état spontané, est de $\mathbf{2} 700$ environ et s'il fallait tenir compte des petites espèces jordaniennes, c'est probablement à plus de 3500 qu'il faudrait porter ce nombre.

Le département du Var qui y confine et a une étendue presque double renferme, d'après le Catalogue (1908) de Albert et Jahandiez, 2123 espèces; enfin dans les départements du Nord de la France, du Nord-Ouest et du Centre on ne compte habituellement que 1000 à 1500 espèces par département.

Le département des Alpes-Maritimes doit sa richesse à la grande variété de ses stations qui s'étendent depuis la mer jusqu'aux montagnes dépassant 3000 mètres d'altitude, à l'existence de terrains calcaires, siliceux et éruptifs anciens, à la température hivernale très chaude du littoral permettant à la plupart des plantes méditerranéennes d'y vivre. Il n'existe pas encore de répertoire des plantes acclimatées. Leur grand nombre tient aux conditions très favorables à l'acclimatation réalisées sur le littoral et aussi à l'existence d'établissements scientifiques (Jardin botanique de la villa Thuret à Ántibes, Jardin de la Mortola en Italie) et d'établissements privés qui ont beaucoup contribué à répandre les plantes qu'ils introduisaient.

$2^{\circ}$ Le département des Alpes-Maritimes est aussi de tous nos départements continentaux celui qui renferme le plus d'espèces endémiques spontanées, localisées exclusivement sur son territoire ou chevauchant sur les contrées avoisinantes. Nous citerons les suivantes :

Ranunculus Canuti Cosson (aujourd'hui rattaché au R.garganicus Ten. d'Italie), Iberis ciliata All., Silene Campanula (All.) Pers., Cytisus Ardoini Fournier, Potentilla Saxifraga Ardoino, Sedum alsinifolium All., Saxifraga florulenta Moretti, S. cochlearis Rchb., S. lantoscana Boiss. et Reut., Asperula hexaphylla 
All,, Leucanthemum discoideum All., Centaurea procumbens Balbis, C. Amilii Briq., Phyteuma Balbisii A. DC., Campanula macrorrhiza Gay, Primula Allionii Lois., Myosotis speluncicola Schott, Orobanche fuliginosa Reuter, Micromeria Piperella Benth., Galeopsis Reuteri Rchb., Euphorbia Saratoi Ardoino, Croeus medius Balbis, Leucoium hyemale DC., Carex Grioletti Ræm.

3. La bande littorale des Alpes maritimes, spécialement entre Nice et Menton-Garavan, présente des conditions de végétation très spéciales sur lesquelles il nous paraît intéressant d'appeler l'attention. Par sa température très élevée en hiver, elle tranche complètement sur les autres parties de la région méditerranéenne continentale au point qu'il serait nécessaire, selon nous, si l'on adopte la nomenclature géobotanique de Flahault pour la France, d'en faire un domaine très spécial que nous proposons de nommer domaine préligurien. Ce domaine comprend la bande côtière située au pied des contreforts des Alpes, qui n'a parfois que quelques centaines de mètres de largeur et rarement plusieurs kilomètres et est fréquemment limitée à l'opposé de la mer par des falaises rocheuses de plusieurs centaines de mètres de hauteur. Ce domaine constitue ce que l'on nomme La Riviera ou Côte d'Azur. Les conditions climatériques y sont très spéciales. Dans les endroits bien abrités, la température n'y descend que très rarement au-dessous de zéro et la neige y est presque inconnue. En outre les rochers tournés du côté de la mer protègent le littoral des vents froids du Nord et par les journées ensoleillées ils emmagasinent de grandes quantités de chaleur. Par une belle journée de soleil, mème en plein hiver, la température au pied de ces rochers est quasi tropicale. C'est une véritable température de serre le jour et, pendant la nuit, par suite de la chaleur emmagasinée, le refroidissement n'est pas très grand. Aussi grâce à cet abri certaines plantes trouvent-elles là des conditions très favorables à leur développement. Le $D^{r}$ Henri Bennett rapporte qu'il ne connait sur la Méditerranée que les environs de Malaga où la végétation soit aussi méridionale, où elle démontre la présence d'une protection aussi grande, d'une chaleur hivernale aussi prononcée. De son côté, le $\mathrm{D}^{\mathrm{r}} \mathrm{E}$. Onimus fait remarquer que les régions les mieux 
abritées par les montagnes, et les plus chaudes autour de la Méditerranée, outre la Côte d'Azur, sont a le Sud de l'Espagne, la province de Malaga, quelques points de la Corse, certaines parties de la Sicile, les environs de Sorrente près de Naples, parce que les montagnes y sont élevées et rapprochées de la mer, ce qui protège contre les vents froids ». Le relief du sol, l'orientation et la proximité des montagnes par rapport avec la mer ont en réalité une influence plus considérable sur le climat que l'éloignement de l'équateur.

Au point de vue de la température, la partie la plus privilégiée du littoral est celle qui va du mont Chauve près Nice au mont Agel près Monaco, c'est-à-dire de Baulieu à Monaco, puis le coin de Garavan entre Menton et le Pont Saint-Louis. La moyenne annuelle de la température à Menton est de $16^{\circ}, 3$; il $y$ a des années où le terme extrême du froid est de $8^{\circ}$ au-dessus de zéro.

Au cours des deux années 1913 et 1916 , nous avons observé semaine par semaine la végétation de cette localité qui présente le fype de la végétation du domaine préligurien.

D'après nos observations, les caractéristiques de la végétation de ce domaine sont les suivantes :

1. Persistance des floraisons' automnales jusqu'au cœur de l'hiver. La gelée ne venant pas arrêter la végétation, un grand nombre de plantes fleurissant tardiyement et dont la végétation s'arrète ailleurs en octobre ou novembre, continuent à donner ici des fleurs pendant l'hiver. Ces fleurs sont du reste normales et donnent des graines.

$2^{\circ}$ Précocité des floraisons printanières. Par suite de la puissante insolation qui se fait sentir en plein hiver sur la Côte d'Azur, bon nombre d'espèces printanières indigènes épanouissent leurs fleurs environ un mois plus tôt que dans les parties du domaine méditerranéen français non abritées par les montagnes ou situées à une certaine altitude. C'est grâce à cette précocité que la Côte d'Azur parvient, en certains points favorisés, à produire en pleine terre des primeurs aussitôt qu'en Algérie ou même aux Canaries. A Menton on récolte en janvier des pommes de terre nouvelles, des pois, des radis, en février et mars des haricots, vers le $1^{\text {er }}$ avril les premières fraises et les 
premières bibasses, au 15 avril les premières tomates; les asperges sont alors en pleine production.

Vers le 15 avril l'écart disparaît dans la flóraison à la côte et dans l'intérieur, car le soleil est plus haut et réchauffe indistinctement tous les points. Par exemple la feuillaison des Platanes commence à Menton dans les premiers jours d'avril; elle n'est que de quelques jours en avance sur la feuillaison des mèmes arbres à Marseille.

$3^{\circ}$ Le domaine préligurien est dans une certaine mesure caractérisé par quelques espèces spéciales. Nous ne citerons que les suivantes :

Papaver prismatifidum Moris, plante de Sardaigne et de Sicile assez répandue dans les terrains cultivés du littoral préligurien.

Laucoium hyemale DC., espèce endémique exclusive à ce domaine et vivant dans les fentes des rochers tournés au Midi et très ensoleillés.

Euphorbia dendroides L. Cet arbuste qui atteint jusquà 2 mètres de hauteur et croît dans les rochers calcaires et les garigues de la zone littorale, est certainement la plante la plus caractéristique de notre domaine préligurien. Dans les endroits bien abrités, très exposés au soleil et presque au niveau de la mer (pied des falaises de Garavan, etc.), certains individus commencent à épanouir leurs fleurs fin janvier; ils sont en pleine floraison au début de mars. Entre Nice et Menton la plante n'est fréquente que dans les rochers et garigues au-dessous de 200 mètres d'altitude; on la voit rarement au-dessus et là l'épanouissement des fleurs a lieu 1 mois ou 1 mois et demi plus tard. C'est ce que l'on constate au Vieux-Eze, dans les rochers et les ruines, à 393 mètres d'altitude.

Cette espèce, assez disséminée en Corse, se rapproche beaucoup par son port et par son mode de végétation de l'Euphorbia balsamifera Ait. des Canaries, de la Mauritanie et du Soudan occidental. Notre plante croît tantôt sur les rochers dénudés exposés au soleil, tantôt dans les bois clairsemés de Pins d'Alep (pineraies du cap Ferrat et du cap Martin), où elle fleurit plus tard et a un aspect un peu différent.

Chamærops humilis L. Ce palmier existait autrefois dans les rochers entre Nice et Monaco où il fut observé par P. de Candolle 
A. Chevalier. - SUR LA FlORE DES ALPES MARITIMES.

en 1808. Il en avait déjà disparu en 1867 d'après Ardoino et ne se rencontre plus qu'à l'état cultivé dans un grand nombre de jardins du littoral.

Deux arbres assez caractéristiques n'existent aussi que là en France à l'état spontané ou subspontané, le Frêne Orne et le Garoubier.

Fraxinus Ornus L. Existe sur les pentes broussailleuses exposées au Midi et dans les éboulis rocheux de la Turbie, à $2 \mathrm{~km}$. environ à l'Ouest de Monaeo, ainsi qu'à Eze dans une station analogue entre le Viaduc du Vieux-Eze et le Tunnel de Saint-Michel. La plante qui se présente dans ces stations sous forme de buissons hauts de 2 à 5 mètres parait bien spontanée.

Ceratonia siliqua L. (Caroubier) est très abondant dans les rochers chauds et ensoleillés, depuis le niveau de la mer jusqu'à 300 ou 400 mètres d'altitude, entre Monaco et Beaulieu, notamment le long de la Moyenne Corniche, dans des endroits tout à fait inaccessibles, où il n'a pu être planté et où il est difficile d'admettre que les graines aient été portées par les animaux. Parfois il est mélangé aux Pins d'Alep. Malgré ces apparences de spontanéité, Alphonse de Candolle et Naudin pensent que le Caroubier est simplement subspontané à la Côte d'Azur, en Espagne et même dans l'Afrique du Nord. Il serait originaire de la Méditerranée orientale et aurait été importé par les invasions arabes.

Une troisième essence ligneuse, l'Ostrya carpinifolia Scop., exclusive aux Alpes maritimes en France et très répandue dans l'arrondissement de Nice au point d'être l'essence dominante de nombreux taillis (monts Farghet, Baudon, Orso, Razet, Mulassier, etc.).

Toutefois on ne saurait la considérer comme caractéristique du domaine préligurien, puisqu'elle s'en éearte considérablement au Nord, au point de foisonner surtout dans la zone montagneuse des Alpes maritimes entre 400 et 1400 mètres d'altitude.

$4^{\circ}$ Le domaine préligurien est surtout remarquable par la flore importée depuis une cinquantaine d'années et qui s'est implantée dans tous les lieux cultivés, au point que cette végétation acclimatée a modifié complètement l'aspect du paysage. Il 
est indispensable que la géographie botanique en tienne compte et nous reviendrons sur ce sujet dans une prochaine étude sur l'acclimatation en France. Certaines acclimatations sont déja anciennes comme celles de l'Oranger et du Citronnier; d'autres remontent à environ un siècle comme le Mandarinier, le Diospyros Kaki, l'Eriobobrya japonica. C'est surtout dans les cinquante dernières années que la flore des pares et jardins de la Riviera s'est enrichie d'espèces exotiques qui donnent au paysage un aspect tropical ou subtropical : Palmiers d'ornement d'une quinzaine d'espèces, Bambous, Eucalyptus, Grevilleas et Casuarinas, arbustes d'ornement fleurissant surtout en hiver, Bougainvillea et Bignoniacées tapissant les murailles, Acacias d'Australie dont il existe déjà une centaine d'espèces ou de variétés horticoles à la Côte d'Azur, surtout l'Acacia dealbata qui commence à se répandre au point d'êtfe commun dans certaines parties de la forêt de l'Estérel, arbustes et arbres fruitiers des contrées tropicales et subtropicales qu'on observe déjà dans certains jardins : Bananiers, Feijoa Sellowiana, Anona Cherimolia, Psidium Cattleyanum, Eugenia myrtifolia, Persea gratissima, Casimiroa edulis.

Je laisse de côté l'Olivier dont la culture est très ancienne et qui atteint une taille exceptionnelle, mais qui existe aussi sur une grande partie du domaine méditerranéen.

Certaines espèces appartenant aux catégories que je viens de citer se sont naturalisées au point de se reneontrer sur les terrains vagues, par exemple le long des talus des chemins de fer. Citons seulement : Mesembrianthemum edule L., Acanthus mollis L., Ricinus communis L., Agave americana, Opuntia Ficus-indica, Gomphocarpus fruticosus R. Br., Erigeron Karwinskianum DC. du Mexique, devenu une des plantes les plus répandues (fréquente sur les murailles à Menton et dans les environs).

Le caractère tropical de cette flore acclimatée est nettement accusé par la rusticité de certaines espèces que nous avons vues au cours de nos voyages en Afrique et en Asie, prospérer dans les régions les plus chaudes des Tropiques, par exemple : Albizzia lophanta, Melia Azedarach, Duranta Plumieri, Jacaranda mimosæfolia, Parkinsonia aculeata, Lanlana Camara, Russelia juncea. 
Du reste, parmi les plantes spontanées dans le domaine préligurien, il en est certaines qui vivent également à l'état spontané dans les pays tropicaux et d'autres qui appartiennent à des genres dont la distribution est surtout tropicale.

A la première catégorie se rapportent l'Imperata cylindrica, cette mauvaise herbe de tous les pays tropicaux du globe, le Cyperus rotundus L., également très répandu dans les contrées chaudes, le Tribulus terrestris, le Cressa cretica et le Lippia nodiflora du Sénégal, le Sorghum halepense Brot., les Andropogon distachyus et A. contortus. Ces deux dernières espèces constituent parfois dans les terrains arides des environs de Menton une formation qui rappelle les savanes des plateaux ferrugineux du Soudan où dominent les mèmes Andropogon ou des espèces affines.

A la deuxième catégorie se rapportent le Myrtus communis qui ressemble à certaines Myrtacées d'Afrique, le Vitex Agnuscastus L., représentant d'un genre qui compte de nombreuses espèces dans les pays tropicaux, le Styrax officinalis L., localisé dans un canton du Var peu éloigné des Alpes maritimes, représentant d'une famille spéciale aux Tropiques; le Plumbago europæa L., qui rappelle le Plumbago zeylanica des régions tropicales. Enfin le Chrozophora tinctoria Juss., le Dipeadi serolinum, le Pancratium maritimum, le Fimbristylis dichotoma Vahl, appartenant à des genres aujourd'hui largement représentés dans l'Afrique du Nord, dans le Sahara et dans l'Afrique tropicale.

Les plantes que nous venons d'énumérer sont évidemment les derniers représentants ou les descendants de la flore tertiaire qui couvrait une partie de l'Europe occidentale pendant l'Éocène et l'Oligocène, à l'époque où vivaient aussi dans ces régions les grands mammifères herbivores tertiaires qui ont émigré en Afrique tropicale. Certaines plantes ont pu persister jusqu'à nous en quelques localités privilégiées du Midi de la France ou elles trouvent aujourd'hui encore des conditions de climat et notamment de chaleur assez semblables à celles qui étaient réalisées à l'époque tertiaire et sans doute comparables à celles qui existent aujourd'hui au Sénégal et dans la zone soudanaise. Cela permet d'expliquer en même temps comment tant de plantes 
tropicales parviennent à s'acclimater aujourd'hui si facilement dans le domaine préligurien des Alpes maritimes.

Ces acclimatations, dans les parcs et les jardins de la Côte d'Azur entraînent peu à peu la disparition de certaines espèces indigènes : les défrichements et les constructions feront peu à peu disparaître les stations où vivent ces plantes. Déjà à Menton on ne trouve plus trace de certaines espèces qu'y avait signalées Ardoino il y a soixante ans. Il y a donc intérêt à noter les stations nouvelles de quelques espèces rares.

\section{Nouvelles localités d'espèces rákes dans les Alpes maritimes.}

Ranunculus Canuti Cosson. - Très commun sur les pentes du mont Baudon, du côté de Sainte-Agnès. Castillon, au-dessous du col de Ségra, vers $900 \mathrm{~m}$. alt.

Anemone stellata L., race à fleurs blanches. - Cap Ferrat : sous les Oliviers près de la crique séparant la presqu'ile principale de la presqu'île de Suinte-Hospice.

Arabis saxatilis All. (=A. nova Vill.). - Sospel, route de Castillon au col Saint-Jean près d'un abreuvoir. Mont Baudon, rochers ombragés. Assez commun.

Viola multicaulis Jord. - Cette espèce est très commune autour de Menton (Castellar, Monti, Sainte-Agnès, Gorbio) et est souvent prise pour $\boldsymbol{V}$. odorata L. qui n'existe qu'auprès des habitations et n'est probablement pas spontané dans la région. - D'après W. Becker, le $V$. multicaulis serait un hybride du $V$. odorata et du $V$. alba Bess. race $V$. Denhardtii Ten. Nous ne pensons pas que ce soit toujours exact, car aux environs de Menton, le $V$. multicaulis est beaucoup plus répandu que les deux parents présumés et il vit souvent en dehors d'eux. En outre il donne des graines fertiles. M. Burnat a déjà signalé dans d'autres genres (Hypericum, Potentilla, Hieracium) l'existente de formes hybrides ressemblant à s'y méprendre à des formes intermédiaires non hybrides et spontanées dans des régions où lun des parents de la plante à origine croisée n'existe pas (Burnat, $\mathrm{Fl}$. Alp. mar., II, p. 27, 1896).

On considère habituellement ces formes (ou races) intermédiaires comme des hybrides fixés, mais on ne possède aucune preuve quils aient eu une hybridation comme origine. Le Viola multicaulis a les fleurs d'un bleu-pervenche, ce qui le distingue de suite du $V$. odorafa. II est très polymorphe et présente certaines variations que nous n'avons constatées ni dans l'un, ni dans Yautre des parents supposés. Par exemple l'éperon est tantôt courbé, tantòt droit et parfois excessivement court. 
Spergularia nicæensis Sarato. - AC. dans les rues à Menton. Abondant dans la cour de la caserne de Roquebrune. Cap Martin.

Spergularia atheniensis Asch. in Schweinfurth. - Roquebrune : baie du cap Martin, du còté du pont de l'Union, C. sur la plage.

Tilia vulgaris Hayne $(=T$. intermedia DC. non Host.). - Très commun le long de la vallée de la Bévera, surtout dans les gorges où son lit est resserré, depuis le bas de Gabane-Vieille (à $6 \mathrm{~km}$. en amont de Moulinet) jusqu'aux environs de Sospel. Bien spontané. Burnat n'admet pas cette espèce comme indigène dans les.Alpes maritimes.

Hypericum hyssopifolium Vill. - Col de Ségra près Castillon.

Vicia nigricans (M. B.) Coss. et Germ. - Mont Orso; pied du mont Rozet.

Pisum elatius M. Bieb. (G. G.). - Castillon et Sainte-Agnès. Est probablement la forme sauvage du Pisum sativum $\mathrm{L}$.

Fragaria collina Ehrh. - Mont Orso, mont Razet.

Pirus amygdaliformis Vill. - Vers $1000 \mathrm{~m}$. d'alt. le long du chemin entre le mont Rozet et le mont Mulacier et dans le col du Traitore au pied du Grammondo. Parait bien spontané quoique non indiqué comme indigène par Burnat.

Malus acerba (DG.) Mérat. - AC. dans les bois : mont Razet, mont Mulacier, Castellar, entre le Grammondo et le Berceau.

Pastinaca urens G. G. - Champs d'oliviers près Castellar.

Smyrnium 0lusatrum L. - Saint-Jean. - cap Ferrat, naturalisé.

Eupatorium glandulosum H. B. K. $(=E$. adenophorum Kunth $=$ $E$. Wendlandi Host. $=E$. trapezoideum Kunth). Abondamment naturalisé sur les terrains dominant le Pont Saint-Louis à Menton et à Monaco, le long du ravin de Sainte-Dévote. Plante de l'Amérique du Sud dont nous devons la détermination à M. Bois.

Carlina acanthifolia All. - AC. dans les montagnes de la région, de $800 \mathrm{a} 1350 \mathrm{~m}$. alt. Le receptacle charnu, large de $6 \mathrm{~cm}$., haut de $3 \mathrm{~cm}$., constitue un légume excellent, non fibreux, de saveur douce et agréable, rappelant les fonds d'artichauts.

Hyoseris radiata L. et Picridium vulgare Desf. - TC. dans la région. On vend ces deux plantes sur le marché de Menton comme " salade-pissenlit ".

Barkhausia bursifolia Spreng. (=Crepis erucifolia Gren.).-AG.à Menton, avenue du Borrigo et près de la gare. N'est peut-être que subspontané.

Hieracium cymosum L. var. canopilosum Arv.-Touv., Rouy. Mont Méras.

Ambrosia maritima L. - Roquebrune. - cap Martin, naturalisé le long de la mer près du Pont de l'Union. 
Pirola chlorantha Sw. - Mont Razet et Grammondo, très commun dans les bois. Trouvé en compagnie de MM. Arbost et Ch. Perret-Gentil. Bois du Farghet! Non encore mentionné à ma connaissance dans les Alpes maritimes.

Phillyrea media L. - Menton : au-dessus de la Gremacide. Commun dans les bruyères du cap Ferrat.

Thymus Serpyllum L., race T. ovatus Mill. - Mont Méras, tout près du col du Farghet, dans les éboulis de rochers vers $1000 \mathrm{~m}$. d'alt.

Thesium montanum Ehrh. - Très commun sur les monts Rozet et Mulacier entre 1100 et $1250 \mathrm{~m}$. d'alt. Commence à fleurir le 10 juin. La plante est abondante en ces deux localités voisines, les seuls points où croisse cette espèce en France. Nous devons à M. Arbost d'avoir pu la récolter en sa compagnie.

Mercurialis annua var. ambigua (L. f.) Duby. - C. dans les jardins secs, les rochers : Menton, Monaco, Beaulieu, etc.

Mercurialis Huetii Hanry. - Mèmes localités, souvent sur les vieux murs et les rochers.

Quercus Cerris L. - Bords de la Bevera en amont de Moulinet, quelques exemplaires çà et là.

Fritillaria involucrata All. - Castillon, près du col de Ségra.

Liliastrum album Link. - Sommet de l'Authion.

Asphodelus fistulosús L. - Eze-sur-Mer.

Ruscus aculeatus L. - Vieux Eze.

Gymnadenia conopea R. Br. race G, alpina (Rehb. f.). Rous. Entre le col de Brous et le mont Farghet.

Gymnadenia pseudoconopéa (Grenier). - Plante à odeur de jacinthe. Bords des champs et prairies près du Vieux Gastellar.

Ophrys litigiosa G. Camus. - Fleurit dès la fin de janvier. Gorbio, Monti, Castellar. AC.

Carex punctata Gaud. - Col du Treitore, au pied du Grammondo, à $1000 \mathrm{~m}$. d'alt.

Carex refracta Schkuhr. - Dans les bois de pins sur le mont Razet, vers $1200 \mathrm{~m}$. alt. Non mentionné dans la flore d'Ardoino,

Anthoxanthum aristatum Boiss. var. nanum (Lloyd) Rouy. Bords de la mer entre le Pont de l'Union et le cap Martin, près Menton.

Asplenium Adiantum-nigrum, s. sp. A. Onopteris L. - Castellar. Vieux murs au village de Sorgio. 


\section{$2 \mathrm{BHL}$ Biodiversity Heritage Library}

Chevalier, Auguste. 1917. "Observations sur la flore des Alpes maritimes." Bulletin de la Société botanique de France 64, 24-34.

https://doi.org/10.1080/00378941.1917.10836002.

View This Item Online: https://www.biodiversitylibrary.org/item/8685

DOI: https://doi.org/10.1080/00378941.1917.10836002

Permalink: https://www.biodiversitylibrary.org/partpdf/159697

\section{Holding Institution}

Missouri Botanical Garden, Peter H. Raven Library

\section{Sponsored by}

Missouri Botanical Garden

\section{Copyright \& Reuse}

Copyright Status: Public domain. The BHL considers that this work is no longer under copyright protection.

This document was created from content at the Biodiversity Heritage Library, the world's largest open access digital library for biodiversity literature and archives. Visit BHL at https://www.biodiversitylibrary.org. 